\title{
Porous Silicon Based Bragg-Grating Resonator for Refractive Index Biosensor
}

\author{
Sourabh SAHU ${ }^{1 *}$, Jalil ALI ${ }^{2}$, Preecha P. YUPAPIN ${ }^{3}$, and Ghanshyam SINGH ${ }^{1}$ \\ ${ }^{1}$ Department of Electronics and Communication Engineering, Malaviya National Institute of Technology Jaipur, India \\ ${ }^{2}$ Laser Centre, IBNU SINA ISIR and Faculty of Science, Universiti Teknologi Malaysia, 81310 Johor Bahru, Malaysia \\ ${ }^{3}$ Computational Optics Group, Advanced Institute of Material Science, and Faculty of Electrical \& Electronics \\ Engineering, Ton Duc Thang University, Ho Chi Minh City, Vietnam \\ *Corresponding author: Sourabh SAHU $\quad$ E-mail: sourabh.ggits@gmail.com
}

\begin{abstract}
In this work, we have evaluated the biosensing capability of the porous silicon (PSi) based sidewall Bragg-grating resonator. The approximation of the quasi-TE mode full vector for the eigenmode calculation is performed using a full vector mode solver. The transmission spectra of the device are evaluated using the transfer matrix method. We have observed a shift in the resonant band for a change in the refractive index of biomaterial in the upper cladding region. The theoretical value of the bulk sensitivity is calculated to be $387.48 \mathrm{~nm} / \mathrm{RIU}$. The device is suitable for biosensing application due to its ability of interacting signal with the infiltrated analytes in the PSi waveguide core.
\end{abstract}

Keywords: Biosensor; evanescent field; eigen mode

Citation: Sourabh SAHU, Jalil ALI, Preecha P. YUPAPIN, and Ghanshyam SINGH, "Porous Silicon Based Bragg-Grating Resonator for Refractive Index Biosensor," Photonic Sensors, 2018, 8(3): 248-254.

\section{Introduction}

The advancement in the photonic field enables the development of compact, precise, and sensitive device for biosensing applications. So far, several sensors are developed for medical-diagnostics, healthcare monitoring, and drug development. Well-known advantages of photonic-based sensors are their inherent capability of providing the immunity to the electromagnetic interference and ease-of-multiplexing for simultaneous detection of the biomolecules [1]. The photonic biosensors for biosensing applications can be of two types, fluorescent and label-free based detectors. The fluorescent technique uses a conventional method of providing the radioactive tag to the targeted biomolecule for their detection [2]. Whereas referring to the label-free approach, the target molecule is detected by attaching biomolecules to the specific bioreceptor on the surface of the biosensor. The attached molecule alters the refractive index of the surface layer that changes the optical characteristics of the propagated signal [1-3]. In the last decade, several label-free biosensors based on the ring resonators, Bragg-grating resonators, slot waveguide, photonic crystal cavities, interferometer, and surface-plasmon-resonance were developed [3-12]. Most of these sensors utilize the planar strip and rib waveguide for device configuration that makes use of the evanescent field

Received: 3 September 2017 / Revised: 29 May 2018

(C) The Author(s) 2018. This article is published with open access at Springerlink.com

DOI: $10.1007 / \mathrm{s} 13320-018-0459-\mathrm{z}$

Article type: Regular 
for detection. Since only small proportion of the evanescent field propagates on the surface of the waveguide, the interaction between the field and biomaterial (analyte) is very low. Recently, many researchers have reported the use of porous material for a waveguide segment that can be an alternative approached for making a highly sensitive biosensor $[13,14]$. Due to the porous nature, a small analyte that is present on the surface can infiltrate in the material and increase interactions with the propagating field [12]. For a photonic biosensor, the porous silicon (PSi) is a preferred material because of the advantages like ease of fabrication using the existing CMOS technology, adaptable geometric plausibility, large surface area $\left(>100 \mathrm{~m}^{2} / \mathrm{cm}^{3}\right)$, and existing surface functionalization science for analyte binding event [13].

The PSi is obtained through the electrochemical etching of the bulk silicon. Accordingly, the concentrated current density modulates the pores dimension of the material that modifies its refractive index. The value of the refractive index is inversely proportional to the dimension of the porosity, and the infiltration of the biomaterial is present in the pores. The sensitivity of the PSi resonant biosensor is $30 \%-50 \%$ more than that of a conventional strip waveguide based devices [15]. Until now, several PSi biosensors based on the multilayer Bragg-reflector [15], microcavities [16], waveguides [17], and Bloch-surface-waves [18] have been proposed. In this work, we have performed the theoretical study for the PSi-based sidewall-grating resonator for a biosensing application. The combined effect of the PSi material and resonator structure for a highly sensitive device is investigated. In this work, we have used a strip waveguide, and modal analysis is performed for the variation of biomaterial on porous silicon for identifying the change in the effective refractive index. The advantage of the proposed sensor is its planar structure. The bends in other resonating devices such as the ring resonator or disk resonator is accompanied by the additional bending losses and requires a larger surface area in the substrate $[5,7]$. Moreover, a planar strip waveguide in the structure is used that requires only single etching process for a fabrication purposes; hence, it significantly reduces the complexity incurred by the multilayer Bragg filters [15].

\section{Effective refractive index approximation of PSi}

The refractive index of the porous silicon lies in between 1 and 3.45 depending on the size of the pores and the infiltrated material. The literature thoroughly covers and provides several dielectric functions such as Maxwell-garnet (MG), Looyenga Landau-Lifshitz (LLL), Bergman's, and Bruggeman model $[14,19]$ to predict the theoretical values of the refractive index of the PSi. The MG model is suitable when the porosity is less than $15 \%$, and the evaluation considers the calculation of the polarization vector of the assumed sphere in the PSi material. The LLL model implements an averaging for dielectric function. Bergman's model is the most complex of all, which considers the dielectric constants, volume fraction, orientation, and geometry of the molecules. The most conventional model used in the literature is the Bruggeman's model that is suitable for the porosity range of 0.32 to 0.66 [14]. It is an extension of the MG model wherein, and it considers both air and silicon molecules between the spheres.

Bruggeman's dielectric function considers the fraction of the analytes, which will be able to penetrate inside the pores for an evaluation of the refractive index. The presence of highly hydraulic resistance between the analyte and the sidewalls of pores refrains the flow of analytes in the pores, so that it can be partially filled. The analytical model for computing refractive index in the near infrared 
range is given as [19]

$$
\begin{aligned}
& (1-p) \frac{n_{\mathrm{si}}^{2}-n_{p}^{2}}{n_{\mathrm{si}}^{2}+2 \cdot n_{p}^{2}}+(p-V) \frac{n_{\text {air }}^{2}-n_{p}^{2}}{n_{\text {air }}^{2}+2 \cdot n_{p}^{2}}+ \\
& V \cdot \frac{n_{\text {analyte }}^{2}-n_{p}^{2}}{n_{\text {analyte }}^{2}+2 \cdot n_{p}^{2}}=0
\end{aligned}
$$

where $p$ is porosity, $n_{\mathrm{si}}, n_{p}, n_{\text {air }}$, and $n_{\text {analyte }}$ are the refractive indices of bulk silicon, $\mathrm{PSi}$, air, and analyte, and $V$ is the fraction of analyte in the pores. In the present work, we have assumed the porosity $p$ $=50 \%$ (suitable for the analyte size of $1 \mathrm{~nm}-10 \mathrm{~nm}$ ), where the refractive index of bulk silicon $\left(n_{\mathrm{Si}}\right)$ and air $\left(n_{\text {air }}\right)$ are 3.45 and 1 , respectively. For finding the change in the refractive index of the porous silicon, we have considered three biomaterials which are blood plasma $\left(n_{\text {analyte }}=1.35\right)$, hemoglobin $\left(n_{\text {analyte }}=\right.$ $1.38)$, and biotin-streptavidin $\left(n_{\text {analyte }}=1.45\right)$ [10]. The value of the refractive index of the PSi for blood plasma, hemoglobin, and biotin-streptavidin is plotted in Fig. 1(a). For analysis, we have considered the value of the refractive index of the PSi for $V=$ $60 \%$ due to its suitability for an approximation of the analyte size.

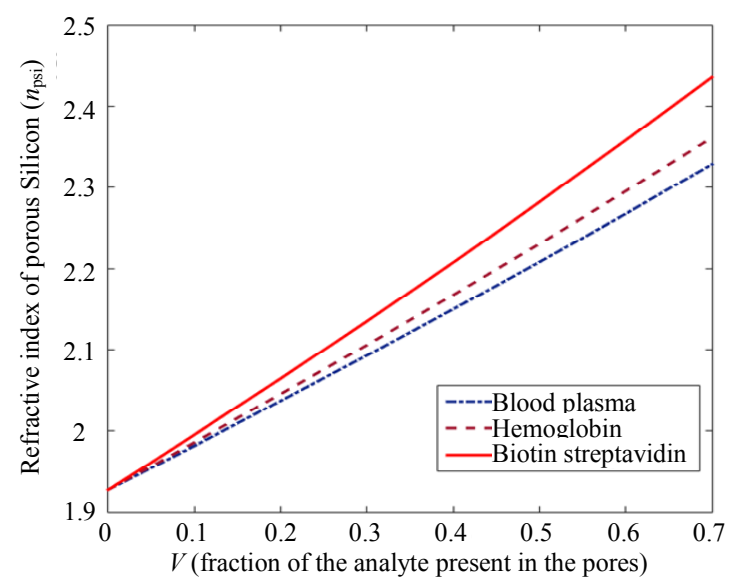

(a)

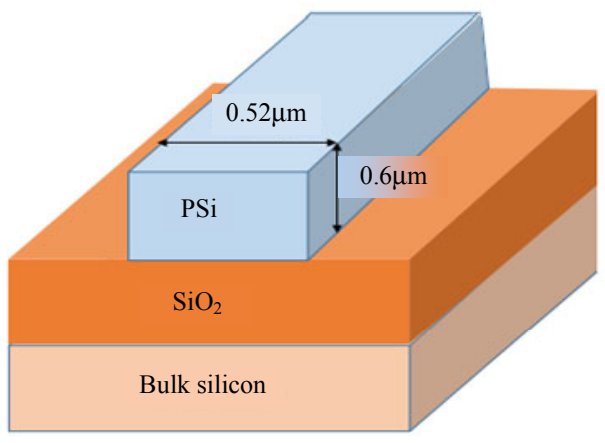

(b)

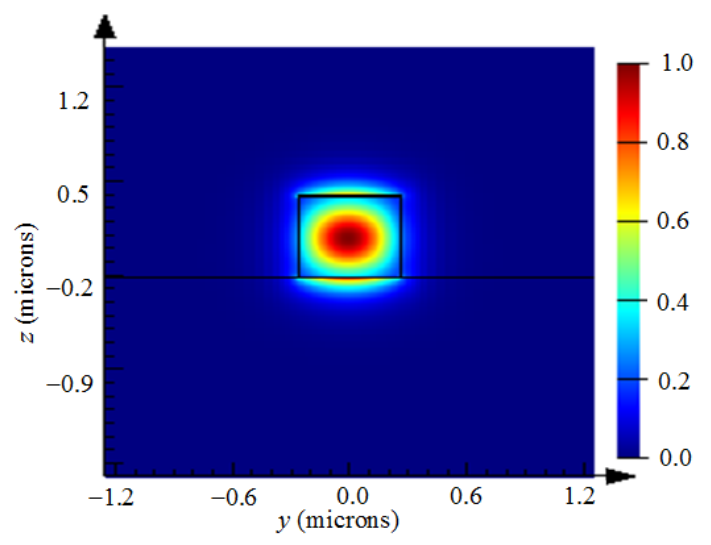

(c)

Fig. 1 Modal analysis of waveguide showing (a) variation in the refractive index of the PSi for $p=50 \%$ for a change in analyte fill fraction " $V$ ", (b) crossectional view, and (c) mode confinement in the strip waveguide.

\section{PSi-based sidewall Bragg-grating biosensor}

Figure 1(b) represents the cross-sectional view of the strip waveguide. The fundamental quasi-TE mode [Fig. 1(c)] is calculated from the solution of modal analysis performed by utilizing the 2-dimensional (2D) full-vector based eigenmode method. The effective refractive index $\left(n_{\text {eff }}\right)$ of the 
PSi is less than that of the bulk silicon that decreases the confinement of the signal in the core of a waveguide. The presence of the signal outside the core increases the signal matter interaction. However, here in this work, it is aimed to design a planar sidewall grating, so for that, a higher value of the $n_{\text {eff }}$ is required [6]. The higher value of the $n_{\text {eff }}$ is the result of an optimization performed by considering the waveguide width and height. The width and height of the core are $520 \mathrm{~nm}$ and $600 \mathrm{~nm}$, respectively.

Bragg-grating resonators are commonly used for sensing applications. Figure 2 shows the schematic of the proposed biosensor. The modulation of the effective refractive index accomplished by altering the waveguide width results in the stop band in the transmission spectrum. The stop band centering at the Bragg wavelength is given as $\lambda_{B}=2 \cdot \Lambda \cdot n_{\text {eff }}$, where $\Lambda$ is the grating period, and $n_{\mathrm{eff}}$ is the effective refractive index. With the aid of an insertion of the additional phase-shift cavity in the periodic structure [Fig. 2(b)], the narrow band is confined into a cavity region, thus a modified the transmission spectrum is expected by providing a sharp resonant peak at the center of the stop band. The evaluation of the transmission spectra has utilized the transfer matrix method in [6]. The microfluidic system for the transporting biomaterial to the device is present on the upper surface of the device that also acts as an upper cladding. The tunable light is coupled to the waveguide at one end, and the output signal is collected at the other end for the spectral analysis.

The parameters of the proposed device are the period of the grating $(\Lambda=600 \mathrm{~nm})$, the corrugation depth $(\Delta W=20 \mathrm{~nm})$, the number of gratings $(N=$ $120)$, and the cavity-length $\left(\Lambda_{c}=600 \mathrm{~nm}\right)$. Figure 3 represents the transmission spectra for a considered biomaterial. The binding event into the specified biomaterials causes a shift in a resonant band. The biosensing parameter can be considered to reflect the sensitivity ( $\left.S=\Delta \lambda / \Delta n_{\text {analyte }}\right)$ that is evaluated through a calculation of a shift in the resonant wavelength for a change in the refractive index of a biomaterial.

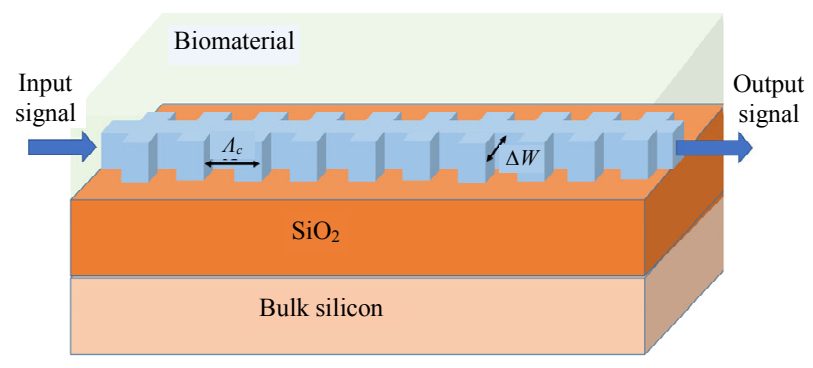

(a)

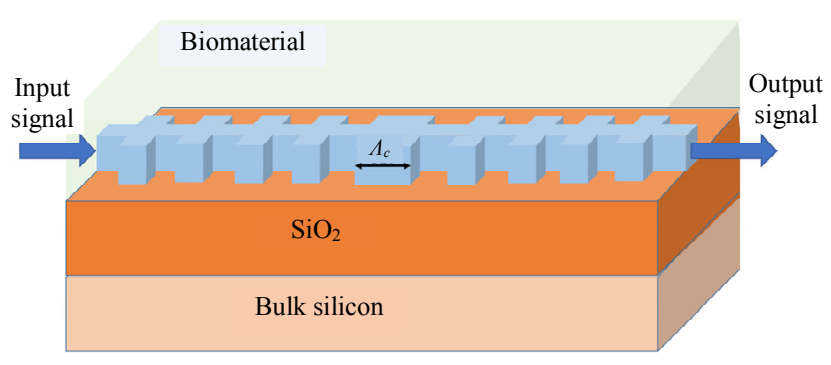

(b)

Fig. 2 Schematic of the Bragg-grating device: (a) without cavity and (b) with cavity $\left(\Lambda_{c}\right)$.

According to Fig. 3, it is observed that the value of transmissivity of the resonant peak reduces for the change in the refractive index of the PSi for a variation of the biomaterial. The change in the biomaterial causes a decrease in the refractive index contrast between the core and the cladding material that results in the decrement of the confinement of the mode in the waveguide and the cavity. Figure 4 represents the linear behavior of the device, hence the calculated value of the sensitivity $\left(\Delta \lambda / \Delta n_{\text {biomaterial }}\right)$ is $387.48 \mathrm{~nm} / \mathrm{RIU}$.

The performance characteristics of some similar biosensors are compared in Table 1 . The sensitivity 
comparison signifies the sure advantage of using the proposed configuration.

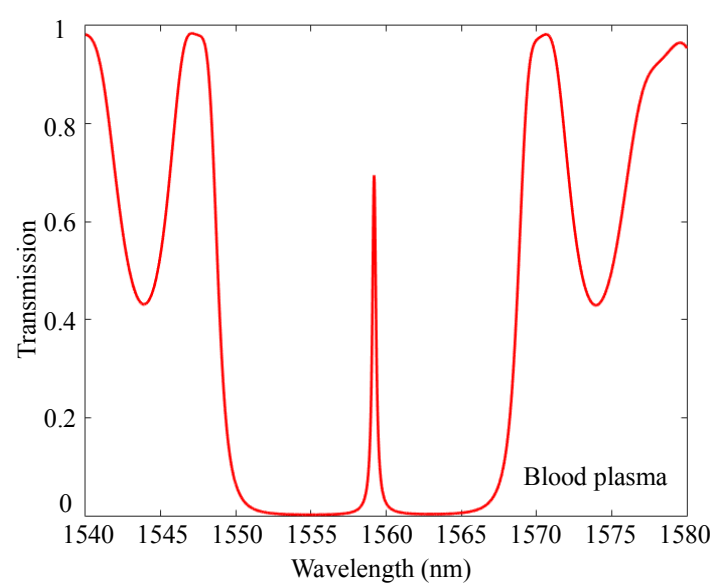

(a)

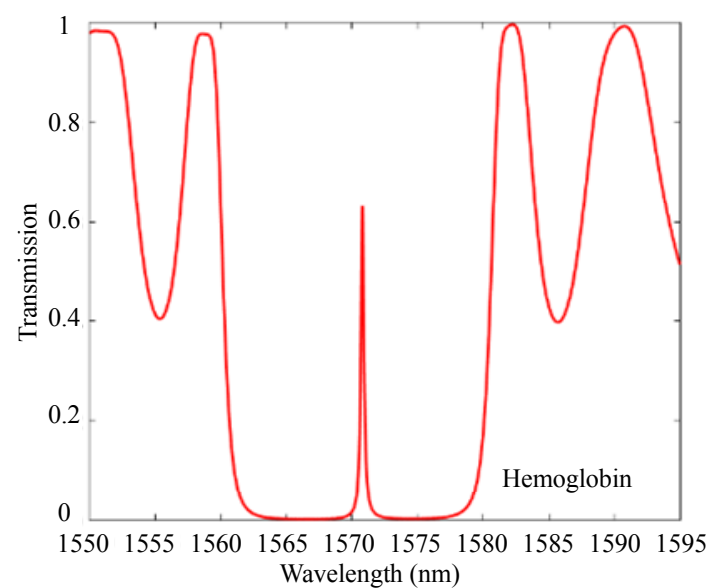

(b)

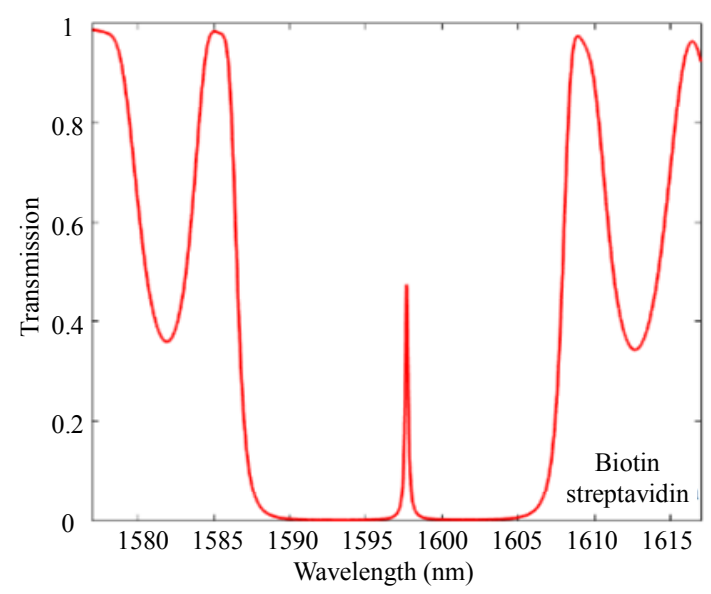

(c)

Fig. 3 Transmission spectra of the biosensor for (a) blood plasma, (b) hemoglobin, and (c) biotin-streptavidin.

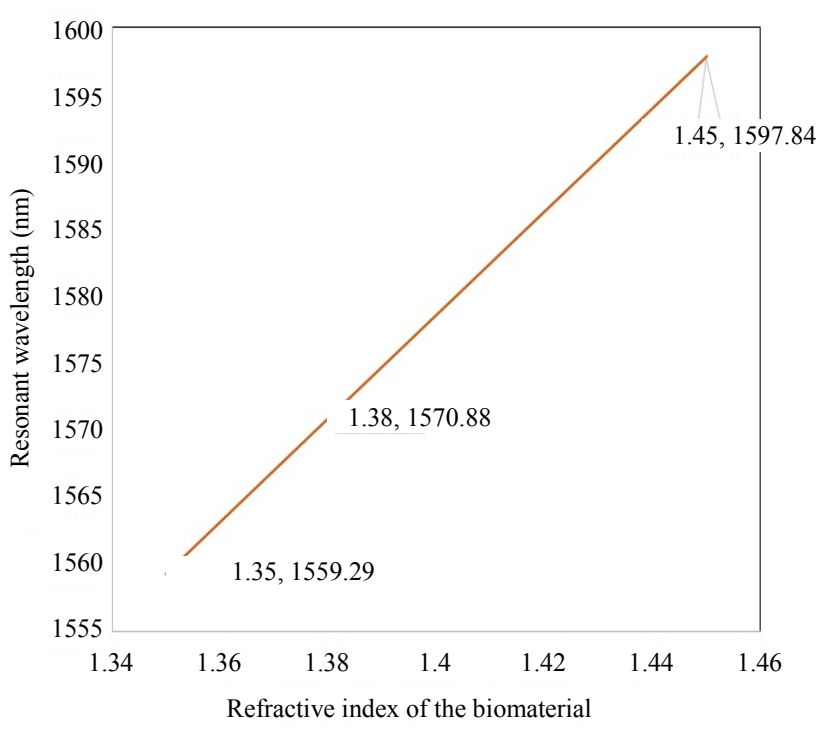

Fig. 4 Variation of the resonant wavelength for a change in the refractive index of the biomaterial.

Table 1 Sensitivity comparison of various biosensors.

\begin{tabular}{cc}
\hline Refractive index of the sensor & Sensitivity (nm/RIU) \\
\hline Ref. [9] & 65.7 \\
Ref. [6] & 291.93 \\
Ref. [20] & 322.96 \\
Proposed sensor & 387.48 \\
\hline
\end{tabular}

\section{Conclusions}

This work is an illustration of the PSi-based sidewall Bragg-grating resonator to act as a refractive index biosensor. The detection is performed by observing the shift in the resonant band for a change in the refractive index of the upper cladding region. The PSi waveguide is designed to operate for the quasi-TE mode, and the confinement of the signal is dependent on the refractive index of the core material. The variation in the refractive index of the PSi by the porosity and fluid fraction in pores is also evaluated. The spectral characteristics of the device are studied through the transfer matrix method. The designed sensor exhibits a bulk sensitivity of $387.48 \mathrm{~nm} / \mathrm{RIU}$. Hence, 
from this work, it is deemed that the device is suitable for biosensing applications.

\section{Acknowledgment}

The authors would like to acknowledge the mutual understanding for joint and collaborative work among researchers from Malaviya National Institute of Technology Jaipur (India), Universiti Teknologi Malaysia, Johor Bahru (Malaysia), and the Ton Duc Thang University, Ho Chi Minh City (Vietnam).

Open Access This article is distributed under the terms of the Creative Commons Attribution 4.0 International License (http://creativecommons.org/licenses/by/4.0/), which permits unrestricted use, distribution, and reproduction in any medium, provided you give appropriate credit to the original author(s) and the source, provide a link to the Creative Commons license, and indicate if changes were made.

\section{References}

[1] S. Balslev, M. Jorgensen, B. Bilenberg, K. B. Mogensen, D. Snakenborg, O. Geschke, et al., "Lab-on-a-chip with integrated optical transducers," Lab on a Chip, 2006, 6(2): 213-217.

[2] X. Fan, I. M. White, S. I. Shopova, H. Zhu, J. D. Suter, and Y. Sun, "Sensitive optical biosensors for unlabeled targets: a review," Analytica Chimica Acta, 2008, 620(1): 8-26.

[3] S. Sahu, K. V. Kozadaev, and G. Singh, "Michelson interferometer based refractive index biosensor," in Proceeding of 13th International Conference on Fiber Optics and Photonics, Kanpur, India, 2016, pp. $1-3$.

[4] W. Yuan, H. P. Ho, C. L. Wong, S. K. Kong, and C. Lin, "Surface plasmon resonance biosensor incorporated in a Michelson interferometer with enhanced sensitivity," IEEE Sensors Journal, 2007, 7(1): 70-73.

[5] D. Irawan, T. Saktioto, J. Ali, and P. Yupapin, "Design of Mach-Zehnder interferometer and ring resonator for biochemical sensing," Photonic Sensors, 2015, 5(1): 12-18.

[6] X. Wang and C. K. Madsen, "Highly sensitive compact refractive index sensor based on phase-shifted sidewall Bragg gratings in slot waveguide," Applied Optics, 2014, 53(1): 96-103.
[7] S. Sahu, J. Ali, and G. Singh, "Refractive index biosensor using sidewall gratings in dual-slot waveguide," Optics Communications, 2017, 402: 408-412.

[8] Y. Cheng and H. Ming, "Review of surface plasmon resonance and localized surface plasmon resonance sensor," Photonic Sensors, 2012, 2(1): 37-49.

[9] S. Olyaee, S. Najafgholinezhad, and H. A. Banaei, "Four-channel label-free photonic crystal biosensor using nanocavity resonators," Photonic Sensors, 2013, 3(3): 231-236.

[10] S. Sahu and G. Singh, "Modeling of phase shift bragg grating biosensor for non-invasive detection of blood components," in Proceeding of IEEE International Conference on Recent Advances and Innovations in Engineering (ICRAIE), Jaipur, India, 2016, pp. 1-3.

[11] M. Sathish and S. Talabattula, "Polarization analysis of an asymmetrically etched rib waveguide coupler for sensing applications," Photonic Sensors, 2013, 3(2): 178-183.

[12] W. C. L. Hopman, P. Pottier, D. Yudistira, J. Van Lith, P. V. Lambeck, R. M. De La Rue, et al., "Quasi-one-dimensional photonic crystal as a compact building-block for refractometric optical sensors," IEEE Journal of Selected Topics in Quantum Electronics, 2005, 11(1): 1116.

[13] Y. Zhao, J. L. Lawrie, K. R. Beavers, P. E. Laibinis, and S. M. Weiss, "Effect of DNA-induced corrosion on passivated porous silicon biosensors," ACS Applied Materials \& Interfaces, 2014, 6(16): 13510-13519.

[14] V. Torres-Costa and R. J. Martín-Palma, "Optical properties of porous silicon materials for biomedical applications," Porous Silicon Biomedical Applications, 2014: 185-222.

[15] P. A. Snow, E. K. Squire, P. S. J. Russell, and L. T. Canham, "Vapor sensing using the optical properties of porous silicon Bragg mirrors," Journal of Applied Physics, 1999, 86(4): 1781-1784.

[16] S. Chan, P. M. Fauchet, Y. Li, L. J. Rothberg, and B. L. Miller, "Porous silicon microcavities for biosensing applications," Physica Status Solidi, 2010, 182(1): 541-546.

[17] G. Rong, A. Najmaie, J. E. Sipe, and S. M. Weiss, "Nanoscale porous silicon waveguide for label-free DNA sensing," Biosensors \& Bioelectronics, 2008, 23(10): 1572-1576. 
[18] X. Wei, J. W. Mares, Y. Gao, D. Li, and S. M. Weiss, "Biomolecule kinetics measurements in flow cell integrated porous silicon waveguides," Biomedical Optics Express, 2012, 3(9): 19932003.

[19] M. Khardani, M. Bouaïcha, and B. Bessaïs, "Bruggeman effective medium approach for modelling optical properties of porous silicon: comparison with experiment," Physica Status Solidi, 2010, 4(6): 19861990.

[20] S. Sahu, J. Ali, P. P. Yupapin, and G. Singh, "Optical biosensor based on a cladding modulated grating waveguide," Optik, 2018, 166: 103-109. 